\section{LITERATURE CITED}

American Ornithologists' Union. 1957. Checklist of North American birds. 5th Edition. p. 585 .

Ferry, J. F. 1910. Birds observed in Saskatchewan during the summer of 1909. Auk 27: 185-204. Cited by Houston and Street (1959).

Francis, C. S. 1950. Lark Bunting. Blue Jay $8(3): 9$.

Gollop, J. B. 1966. Saskatoon Bird Review $1(3): 23$.

Gollop, J. B., J. A. Slimmon, and R. V. Folker, 1966. Some 1965 bird records for the Saskatoon district. Blue Jay 24 :76-78.
Houston, C. S. and M. G. Street. 1959. The birds of the Saskatchewan River. Saskatche. wan Natural History Society. p. 186.

Kvinge, H. 1960. Influx of Lark Buntings at Hawarden. Blue Jay $18: 118$.

Pearson, T. G. et al, editors. 1917. Birds of America. Garden City Publ. Co.: New York. Part III, p. 76 .

Roy, J. F. 1960. Birds of special interest in the Saskatoon area, spring, 1960. Blue Jay $18: 117-118$.

Roy, J. F. 1964. Birds of the Elbow. Saskatchewan Natural History Society. p. 30 .

\title{
RECENT OBSERVATIONS OF THE FIELD SPARROW IN MANITOBA
}

\author{
by Martin McNicholl, 1281 Valour Road, Winnipeg
}

In the early afternoon of October 3, 1965, when I was carrying out a routine check of the birds in Brookside Cemetery, Winnipeg, I was surprised to hear a bird in song. I could not recall ever having heard the song before, but believed it to be one of the small sparrows. Anxious to identify the author of a bird-song in October, I immediately headed towards the sound. My suspicions of a small sparrow were further strengthened on finding that the song came from an area of the cemetery where native prairie with tall grasses still remains almost untouched except for a fairly large brush pile and a few small trees. This is a favorite spot of migrant sparrows.

On spotting the singer, I at first thought it was a Tree Sparrow (Spizella arborea). Suddenly, however, I realized that the bird had a distinctly pink bill. Suspecting a Field Sparrow (Spizella pusilla), I noted the following features through $8 \times 42$ binoculars: "pink bill, clear greyish breast with no breast spot, whitish wing bars, greyish-brown nape, reddish in wings and upper regions (and cap), and whitish throat." The lack of a breast spot was not entirely convincing, as I have seen Tree Sparrows in the fall without this very characteristic feature.
Moreover, I could not be positive that the bird had a white eye-ring, although I thought it did. Suddenly, however, the bird moved closer to me, erabling me to make out the eye-ring clearly. I was then convinced that I was watching a Field Sparrow. My conclusion was verified later in the afternoon by Mr. Vere Scott.

This observation again opened the question of the status of the Field Sparrow in Manitoba. E. E. Thompson (=Ernest Thompson Seton) never recorded it personally, but in his Birds of Manitoba (1890) he recorded the following statements: "Very rare summer resident. Red River Settlement: Breeding (D. Gunn) Winnipeg: Summer resident; tolerably common (Hine). Have seen it west of Winnipeg (R. H. Hunter). Qu'Appelle: common summer resident; breeds; arrives April 15 (Guernsey)."

The above observations were believed by John Macoun (Macoun and Macoun, 1909) to be mostly, if not completely, in error; Macoun was convinced that these observers were probably referring to the Clay-colored Sparrow (Spizella pallida),.. which, although abundant in southern Manitoba, was not mentioned by Guernsey, Hunter or Hine.

The Field Sparrow was not definitely recorded in Manitoba until 
June 30, 1960, when Harold V. Hosford found a nest in Charleswood (at Winnipeg). The nest contained one Field Sparrow egg and one egg of the Brown - headed Cowbird (Molothrus ater). The cowbird egg hatched on July 6 , and the nestling was fed until July 15. The Field Sparrow egg did not hatch. It was collected by Richard W. Sutton on July 14, and was found to contain a dessicated embryo about six days old. The Field Sparrow was definitely identified by Hosford on July 3, and was confirmed by Angus H. Shortt on July 6, and Sutton on July 14 (Hosford, 1962; Mossop, 1960).

Although no details seem to be available of an observation of a Field Sparrow at Wawanesa on May 14, 1961, by Ed Robinson, his longstanding reputation leaves little doubt as to the validity of this record (Mossop, 1961).

On September 20, 1964, Roy Simmons was surprised to discover a Field Sparrow in his banding traps in St. Vital (at Winnipeg). This startling find was verified by Angus Shortt. To add to the excitement, a second Field Sparrow entered his traps on the same day. A third Field Sparrow was trapped by Simmons on September 29, 1965 (Hosford, 1964 and 1965) about a week or two before we observed the bird in Brookside Cemetery.

This sudden series of observations in the first half of this decade raises a number of questions. Has this species always occurred in Manitoba and simply been overlooked? This seems unlikely unless it has been here in very small numbers. Possibly the species has fluctuated in numbers over the years. If some of the early records are valid, then perhaps this species was more common in Manitoba before the turn of the century. Thinking that perhaps abservers in Minnesota and North Dakota might have recorded a northern range extension, I wrote to Robert B. Janssen and Robert E. Stewart for information on this species; both reported an absence of evidence for this situation.
The latest A.O.U. Check-list (1957) gives the nearest breeding record to Manitoba (of the race Spizella pusilla pusilla) as Nisswa in central Minnesota, and (of the race S.p. arevacea) Charlson and Minnewauken in northern North Dakota.

The only record for Saskatchewan supposedly is of one banded on October 6, 1946 at Burnham (in the extreme southwestern part of the province) by Arthur Ward (1952), but this record is believed to be uncertain (pers. corres., C. S. Huston to R. W. Nero, 1958), and this species is not listed for Saskatchewan. However, as the Field Sparrow breeds in northern Montana and North Dakota, observers in southern Saskatchewan should watch for it.

Both Mr. Hosford and I felt that the birds we saw resembled the Tree Sparrow much more strikingly than the Chipping Sparrow (S. passerina); and that the white eye-ring, although very clear at close range, is a more difficult field mark to see than other features, e.g., the pink bill which is readily seen.

I would like to thank Mr. Harold V. Hosford, Mr. Robert B. Janssen, Dr. Robert W. Nero, Mr. Roy Simmons, and Dr. Robert E. Stewart for information they provided for this note.

\section{LITERATURE CITED}

American Ornithologists' Union. 1957. Checklist of North American birds. Fifth ed. Baltimore. $691 \mathrm{pp}$.

Hosford, H. V. 1962. Breeding record of the Field Sparrow in Manitoba. Can. Field-Nat., $76: 178$.

Hosford, H. V. 1964. Rare Field Sparrow unexpected visitor. "Wild Wings", The Winnipeg Tribune, October 5, 1964.

Hosford, H. V. 1965. Never offer a drink to a bird. "Wild Wings", The Winnipeg Tribune, October 23, 1965.

Macoun, J.. and J. M. Macoun. 1909. Catalogue of Canadian birds. Can. Dept. of Mines, Geol. Surv. Branch, Ottawa.-No. 973, pp. 1-761.

Mossop, H. 1960. Field Sparrow nest. "Chickadee Notes No. 288. The Winnipeg Free Press.

Mossop, H. 1961. Migration reports. "Chickadee Notes" No. 332. The Winnipeg Free Press.

Thompson, E. E. 1890. The Birds of Manitoba. Proc. U.S. Natl. Mus., No. 841, Vol. 13, pp. 457-643.

Ward, A. 1952. Notes from a bird bander. Blue Jay, $10(4): 9,13$. 


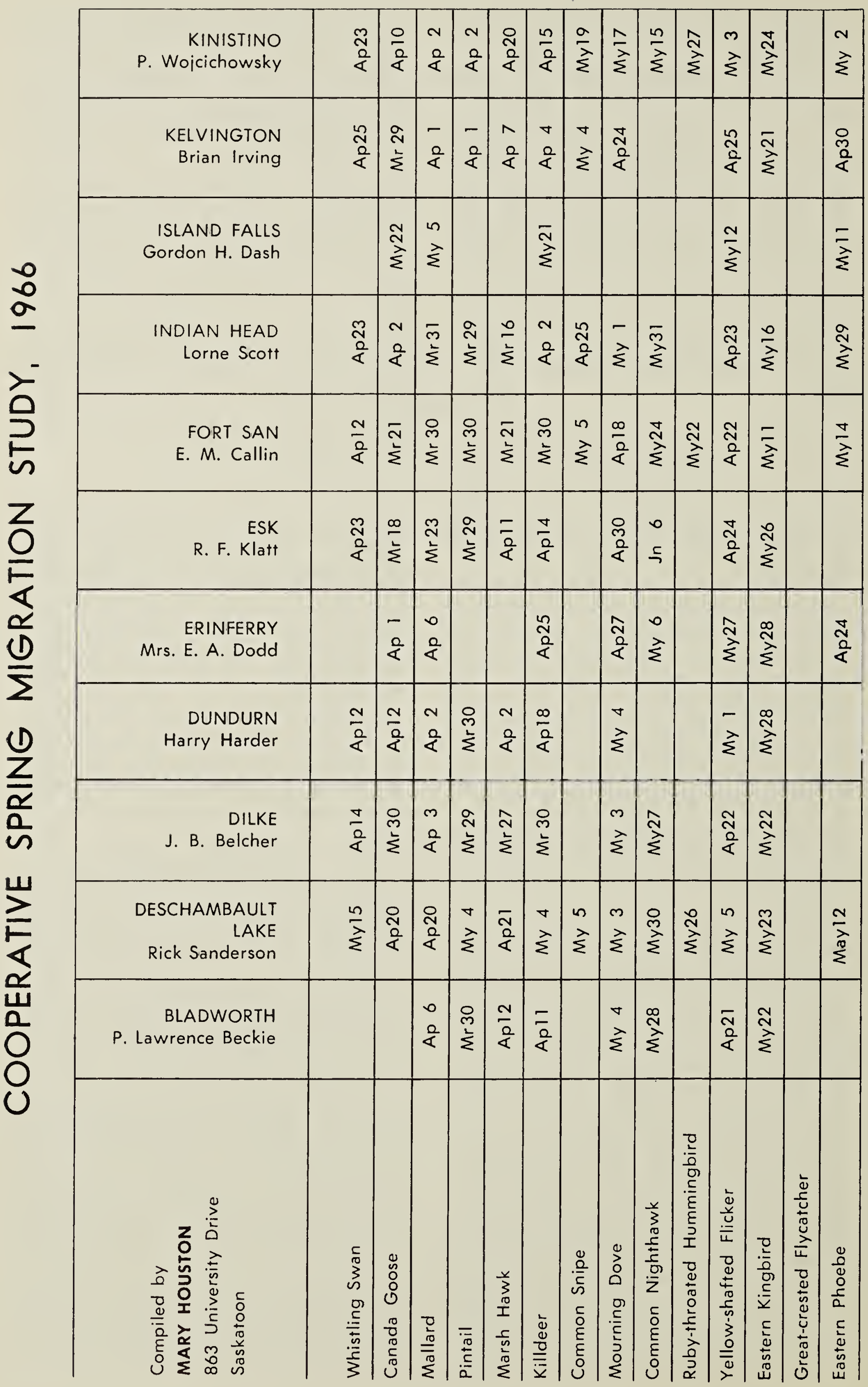




\begin{tabular}{|c|c|c|c|c|c|c|c|c|c|c|c|c|c|c|c|c|c|}
\hline 초 & $\stackrel{\stackrel{N}{ָ}}{\stackrel{\Sigma}{\Sigma}}$ & $\stackrel{\stackrel{n}{N}}{\stackrel{N}{\Sigma}}$ & $\stackrel{\stackrel{R}{ָ}}{\stackrel{\Sigma}{\Sigma}}$ & $\stackrel{\text { N }}{\stackrel{\text { }}{\Sigma}}$ & $\begin{array}{l}\text { N } \\
\text { 之े }\end{array}$ & & $\stackrel{⿱ 亠 乂}{\stackrel{N}{\Sigma}}$ & $\frac{\nabla}{i}$ & $\begin{array}{l}\text { m } \\
\text { 단 }\end{array}$ & సั & 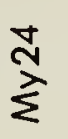 & $\stackrel{\text { NO}}{\stackrel{\Sigma}{\Sigma}}$ & $\begin{array}{l}m \\
\simeq\end{array}$ & 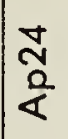 & $\stackrel{n}{i}$ & $\frac{\nabla}{\grave{\Sigma}}$ & $\frac{0}{\lambda}$ \\
\hline$\hat{n}^{\lambda}$ & & $\begin{array}{l}\stackrel{\text { N }}{\Sigma} \\
\text { 之े }\end{array}$ & $\underset{\text { No }}{\stackrel{\text { L }}{\hat{~}}}$ & 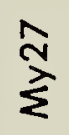 & & & $\frac{m}{\grave{k}}$ & 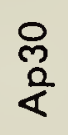 & & సু & $\underset{\mathcal{N}}{\bar{\Sigma}}$ & & 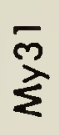 & $\begin{array}{l}m \\
\frac{0}{\alpha}\end{array}$ & 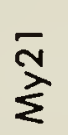 & $\begin{array}{l}n \\
\sum\end{array}$ & $\hat{\Sigma}^{2}$ \\
\hline$\underset{\sum}{\bar{N}}$ & & $\frac{a}{2}$ & & & $\begin{array}{l}m \\
ᄃ\end{array}$ & $\begin{array}{l}m \\
\subseteq\end{array}$ & $\underset{\text { N }}{\stackrel{\text { }}{\Sigma}}$ & $\frac{\nabla}{\grave{z}}$ & & $\frac{ \pm}{\grave{z}}$ & & & & $\frac{1}{\frac{0}{\alpha}}$ & $\frac{n}{\frac{n}{2}}$ & $\frac{n}{2}$ & ָָ \\
\hline
\end{tabular}

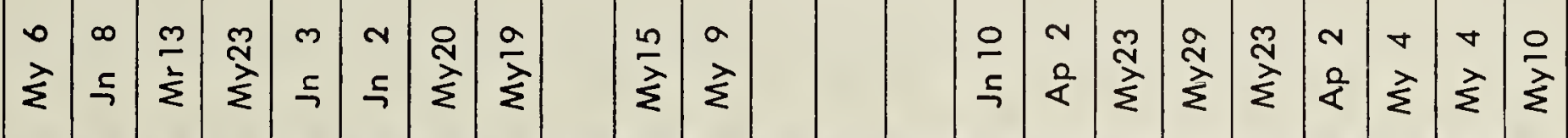

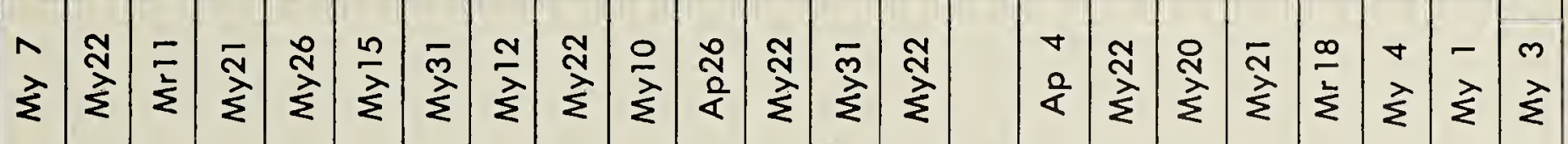

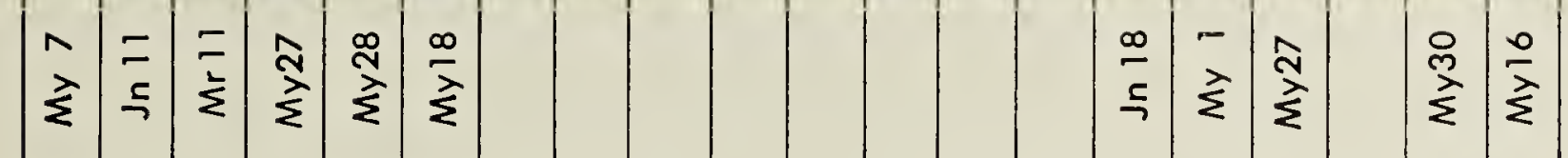

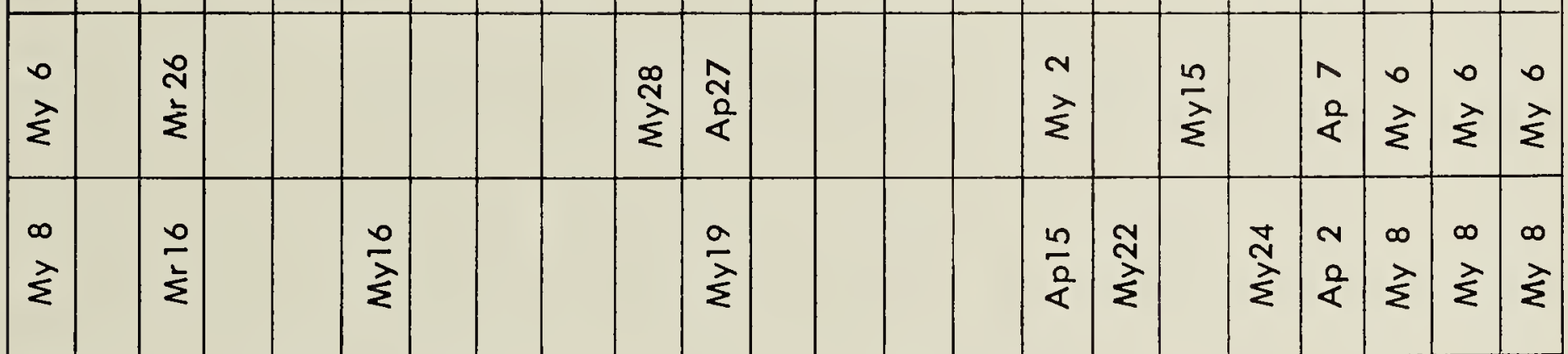

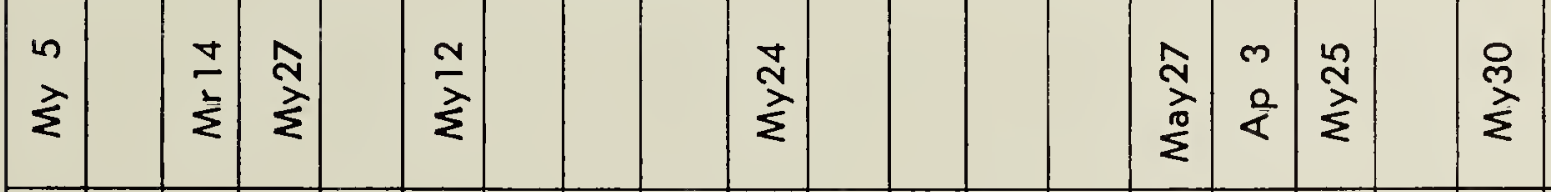

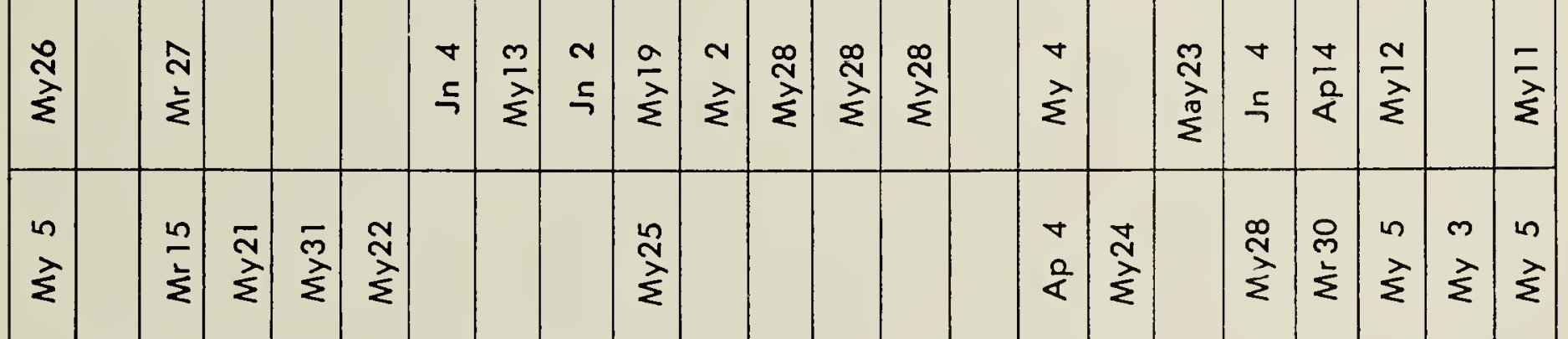

$\sqrt{1}$ 


\begin{tabular}{|c|c|c|c|c|c|c|c|c|c|c|c|c|c|c|}
\hline $\begin{array}{l}\text { YELLOW GRASS } \\
\text { Mrs. R. Peterson }\end{array}$ & $\begin{array}{l}\infty \\
\stackrel{2}{4}\end{array}$ & $\frac{2}{8}$ & $\frac{2}{8}$ & $\begin{array}{l}n \\
2 \\
\alpha\end{array}$ & $\overline{\bar{\alpha}}$ & 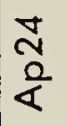 & & 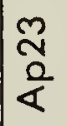 & & & $\sum_{i}^{\infty}$ & $\mathfrak{N}_{\substack{i \\
⿱ 亠 乂}}$ & $\sum_{\Sigma}^{\bar{N}}$ & 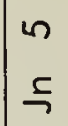 \\
\hline $\begin{array}{l}\text { YELLOW CREEK } \\
\text { Derwent Mazur }\end{array}$ & $\frac{\infty}{\frac{0}{4}}$ & $\begin{array}{l}\hat{N} \\
\stackrel{\Sigma}{\Sigma}\end{array}$ & $\begin{array}{l}m \\
\frac{0}{\alpha}\end{array}$ & $\frac{m}{2}$ & $\begin{array}{l}\frac{\alpha}{2} \\
0\end{array}$ & $\frac{1}{2}$ & $\vec{z}$ & $\frac{\nabla}{2}$ & $\begin{array}{l}\nabla \\
\text { ᄃ }\end{array}$ & $\stackrel{2}{\sim}$ & 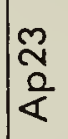 & 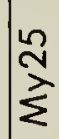 & & ì \\
\hline $\begin{array}{l}\text { YELLOW CREEK } \\
\text { Bohdan Pylypec }\end{array}$ & & $\frac{-}{2}$ & $\frac{2}{4}$ & $\begin{array}{l}n \\
2 \\
8\end{array}$ & 产 & $\frac{n}{\frac{0}{2}}$ & 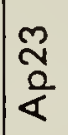 & $\sum_{\Sigma}^{0}$ & $\begin{array}{l}\circ \\
\text { ᄃ }\end{array}$ & Ǹ & 竎 & $\underset{N}{N}$ & & - \\
\hline
\end{tabular}

$\frac{Z}{2}$

$\frac{\text { D }}{\frac{2}{n}}$

\begin{tabular}{|c|c|c|c|c|c|c|c|c|c|c|c|c|c|c|}
\hline $\begin{array}{l}\text { SPIRIT LAKE } \\
\text { Wm. Anaka }\end{array}$ & $\frac{\text { స్ }}{\frac{\alpha}{4}}$ & $\frac{0}{\frac{1}{\Sigma}}$ & $\mid \begin{array}{l}\infty \\
8 \\
8\end{array}$ & $\begin{array}{l}1 \\
8\end{array}$ & $\begin{array}{l}N \\
\& \\
\&\end{array}$ & $\begin{array}{l}a \\
\&\end{array}$ & 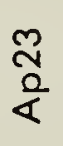 & $\mid \begin{array}{l}\frac{\alpha}{2} \\
\frac{8}{2} \\
0\end{array}$ & $\stackrel{1}{\leftrightharpoons}$ & $m$ & 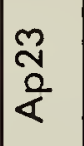 & 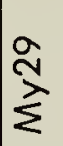 & $\stackrel{\infty}{i}$ & $\frac{d}{2}$ \\
\hline $\begin{array}{l}\text { SKULL CREEK } \\
\text { S. A. Mann }\end{array}$ & $\stackrel{8}{2}$ & $\frac{\pi}{\frac{\pi}{2}}$ & 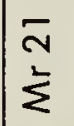 & 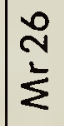 & $\stackrel{N}{\Sigma}$ & 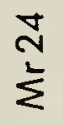 & $\frac{\sqrt{6}}{\sqrt[s]{4}}$ & \begin{tabular}{l}
2 \\
\multirow{2}{\alpha}{} \\
$\frac{2}{<}$
\end{tabular} & & & 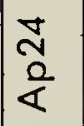 & 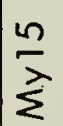 & & \\
\hline $\begin{array}{r}\text { SHEHO } \\
\text { William Niven }\end{array}$ & $\frac{\text { ลี }}{<}$ & $\underset{\substack{n \\
n}}{4}$ & $\begin{array}{l}n \\
2 \\
2\end{array}$ & $\begin{array}{l}1 \\
\frac{8}{2}\end{array}$ & 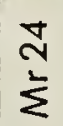 & $\frac{\pi}{\frac{2}{2}}$ & ${ }_{i}^{N}$ & 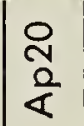 & $\begin{array}{l}8 \\
\text { s }\end{array}$ & $\stackrel{0}{\circ}$ & $\frac{0}{\frac{0}{8}}$ & 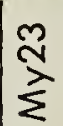 & & \\
\hline
\end{tabular}

J. B. Gollop \& N.H.S.

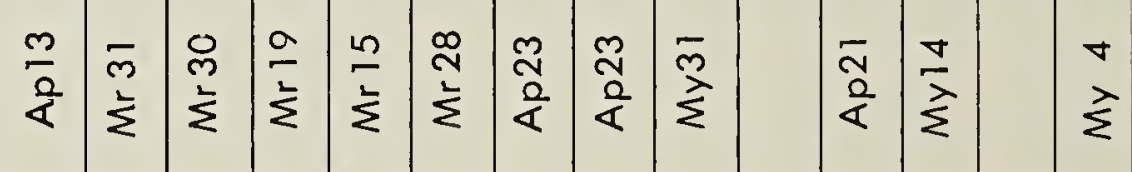

REGINA

R.N.H.S.

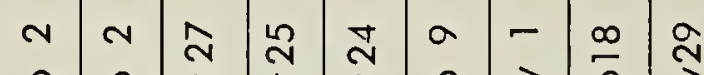

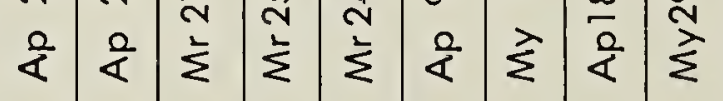

$\underset{\substack{\alpha \\ \frac{\alpha}{\alpha}}}{\bar{N}}$

$\stackrel{2}{\sum}$

NIPAWIN

M. G. Street

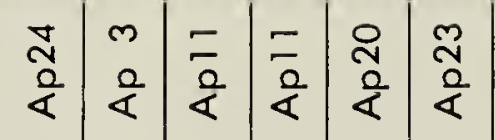

年

$\sum^{\infty}$

MOOSE JAW

M.J. Nat. Hist. Soc.

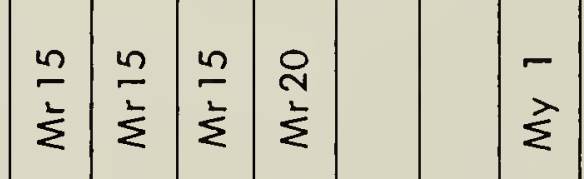

LUCKY LAKE

Mrs. E. C. Boon

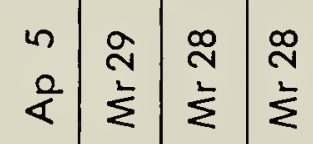

$\nabla$

운

$\stackrel{5}{\neg}$

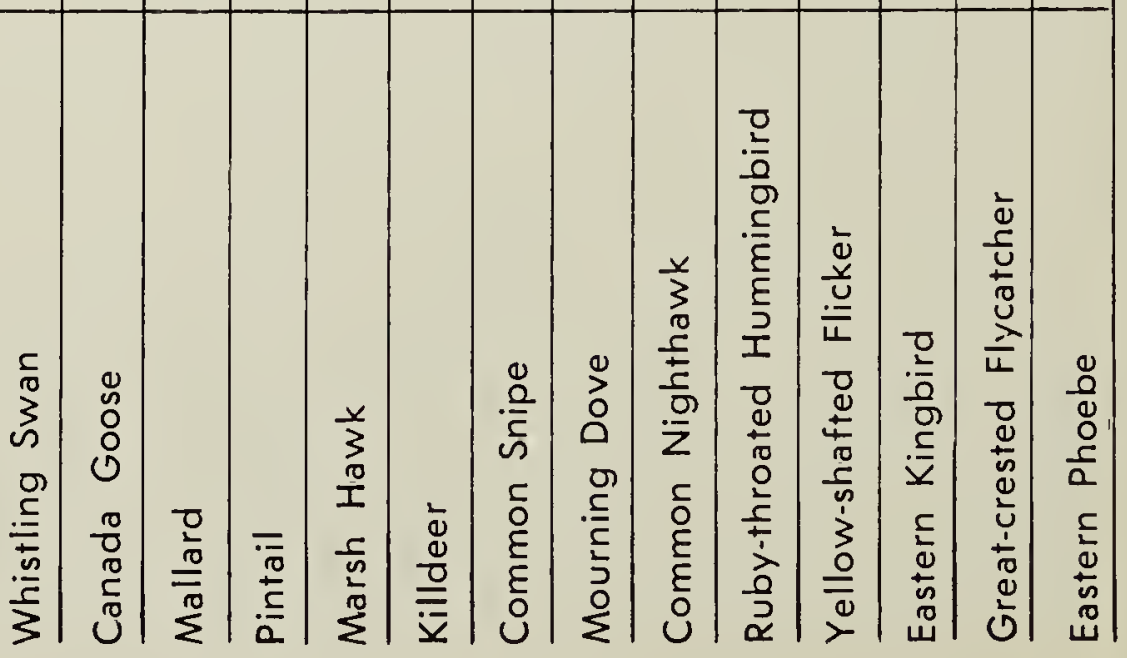




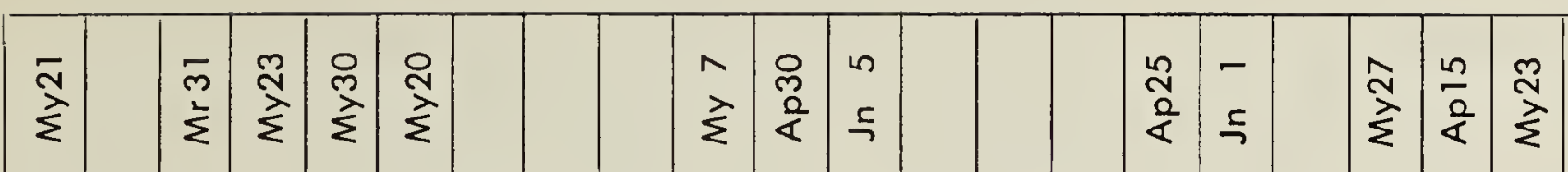

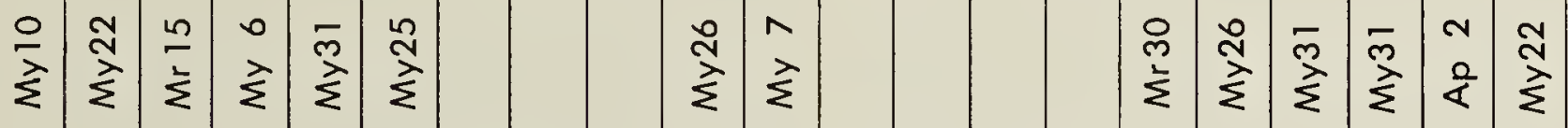

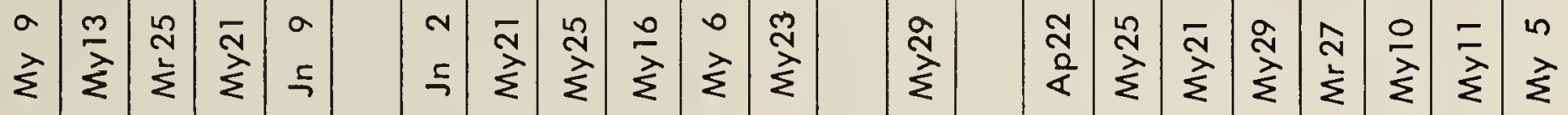

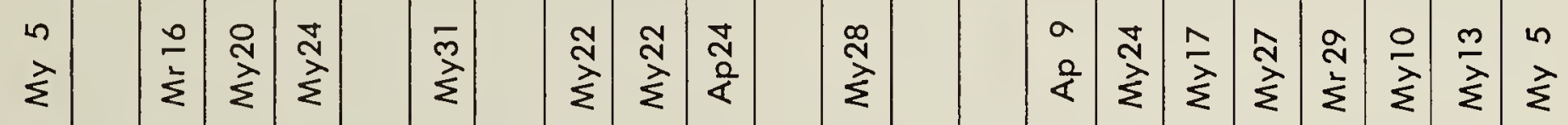

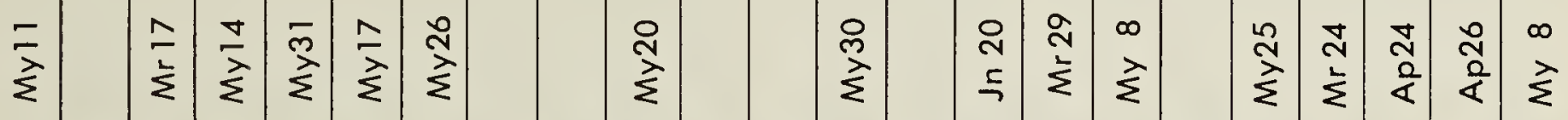

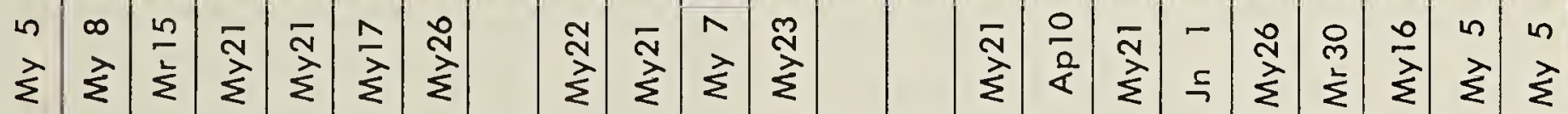

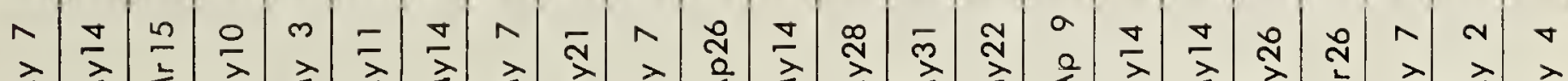

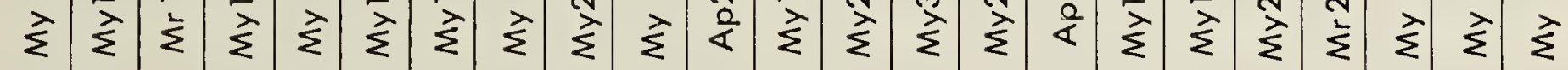

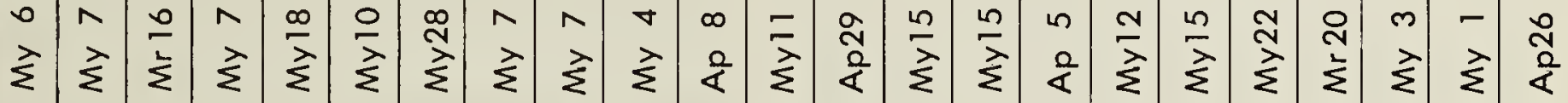

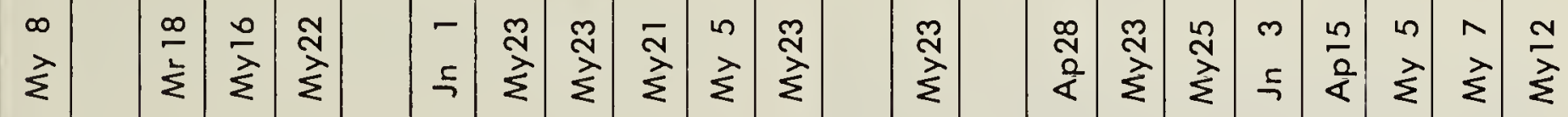

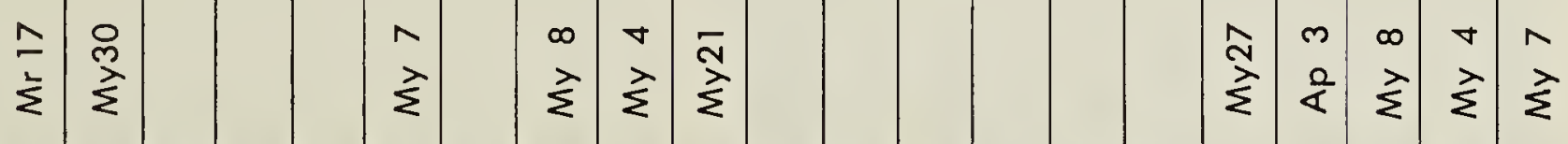

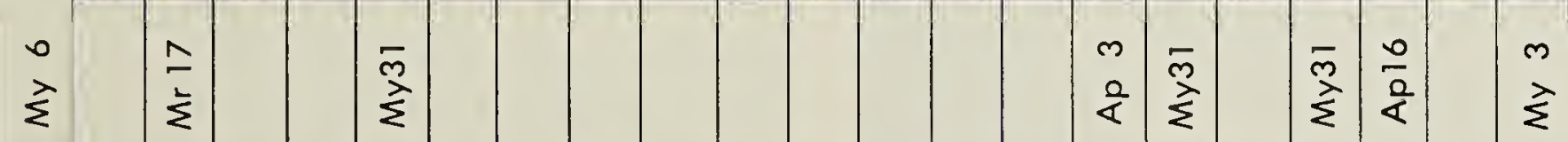

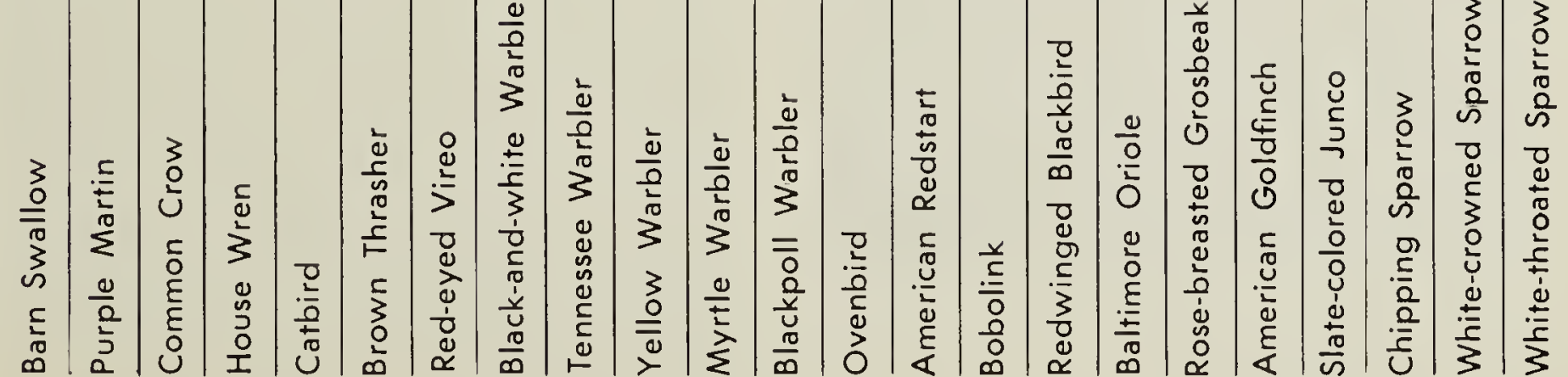

